Relations industrielles

Industrial Relations

\title{
Travail d'équipe dans l'industrie
} Comités patronaux-ouvriers de production

Volume 6, numéro 4, septembre 1951

URI : https://id.erudit.org/iderudit/1023137ar

DOI : https://doi.org/10.7202/1023137ar

Aller au sommaire du numéro

Éditeur(s)

Département des relations industrielles de l’Université Laval

ISSN

0034-379X (imprimé)

1703-8138 (numérique)

Découvrir la revue

Citer cet article

(1951). Travail d'équipe dans l'industrie : comités patronaux-ouvriers de production. Relations industrielles / Industrial Relations, 6(4), 117-122.

https://doi.org/10.7202/1023137ar

Tous droits réservés (C Département des relations industrielles de l’Université Laval, 1951
Ce document est protégé par la loi sur le droit d'auteur. L’utilisation des services d'Érudit (y compris la reproduction) est assujettie à sa politique d'utilisation que vous pouvez consulter en ligne.

https://apropos.erudit.org/fr/usagers/politique-dutilisation/ 
sentences arbitrales et l'article qui complète celui-ci, nous reproduirons textuellement ces deux articles:
\& Art. 16. - L'accord de la conciliation ainsi que les sentences arbitrales sont obligatoires. Ils produisent effet. en principe, à dater du jour du dépôt de la requête aux fins de conciliation. La sentence arbitrale est notifiée aux par- ties dans les vingt-quatre heures de sa date par les soins de l'arbitre. Cette no- tification est faite par lettre recomman- dée avec accusé de réception. La mi- nute de laccord ou de la sentence est, dans le même délai, déposée au secré- tariat du conseil de prud'hommes, à défaut de conseil de prud'hommes, au greffe de la justice de paix du lieu où est déposée la convention collective ou l'un des accords prévus aux articles 31 du livre ler du Códe du Travail ou 21 de la présente loi ou, à défaut de con- vention et d'accord, du lieu où ils ont été rendus. Ce dépôt est effectué, à frais communs, pour l'accord de con- ciliation, aux soins de la partie la plus diligente, et pour la sentence arbitrale, par l'arbitre. Par le seul fait de ce dé- pôt, l'accord ou la sentence a force exécutoire. Les arrêts et sentences de la Cour supérieure d'arbitrage seront pu- bliés tous les trois mois au Journal offi- ciel. »

* Art. 17. - Lorsqu'un accord de conciliation ou une sentence arbitrale devenue exécutoire portent sur l'interprétation des clauses d'une convention collective existante, sur les salaires ou sur les conditions de travail, cet accord ou cette sentence, sous réserve du dépôt prévu à l'article 16 ci-dessus, produira les effets d'une convention collective de travail Si l'accord ou la sentence est intervenu en vue de régler un conflit survenu dans une branche d'activité ayant fait l'objet d'une convention collective étendue en application de l'article 31-j du livre ler du Code du Travail, cet accord ou cette sentence devra, à la demande des organisations signataires de la convention collective étendue, faire l'ob'et d'un arrêté d'extension pris conformément aux dispositions des articles 31-j, 31-k et 31-1 du livre 1er du Code du Travail. Cet arrêté pourra être rapporté dans les conditions prévues au deuxième alinéa de l'article $31-m$ du livre ler du Code du Travail. Les dispositions de la section VIII du chapitre IV bis du titre II du livre ler du Code du Travail s'appliquent aux accords de conciliation, et aux sentences arbitrales avant fait l'obiet d'un arrêté d'extension. Tous actes faits en exécution des dispositions du présent titre sont dispensés du membre et enregistrés gratis. »

\section{Travail d'équipe dans l'industrie *}

\section{Comités patronaux-ouvriers de production}

Un film, ayant pour objet la promotion de relations harmonieuses entre les employeurs et les travailleurs, a été montré en primeur récemment dans un cinéma d'Ottawa.

Les invitations à cette première venaient de l'Honorable Milton F. Gregg, ministre fédéral du Travail, commanditaire de cet intéressant film documentaire.

Le film «Travail d'équipe, passé et présent » fait voir le développement de la coopération parmi les hommes dès les temps anciens en démontrant ses avantages et le rôle qu'elle a joué dans l'avénement de la civilisation. Il montre qu'avec l'accroissement

* Article rédigé en collaboration par des officiers du ministère fédéral du Travail. constant des entreprises industrielles, le travailleur avait pratiquement perdu toute initiative du fait qu'il n'y trouvait aucun moyen d'expression de ses idées et que ce sentiment de frustration l'avait rendu mécontent de son sort. Il signale aussi, comment les ouvriers se sont graduellement tournés vers le syndicalisme en nombre croissant, jusqu'à ce qu'il soit devenu une force capable de s'opposer aux patrons et à la direction, situation plus apte à créer des conflits qu'à promouvoir une société de partenaires.

A ce point, le film nous le décrit, intervient l'idée de coopération et de consultation entre les deux participants dans l'industrie. L'idée n'était 
pas nouvelle. Et le film rappelle qu'on la retrouve au commencement de l'histoire de l'homme, mais que le céveloppement rapide de l'industrialisation l'avait fait oublier. Ce qui est nouveau, c'est l'application de cette idée. Et la dernière partie du film est consacrée à l'encouragement de cette nouvelle méthode.

Et cette méthode, les comités ouvriers-patronaux de production, dont l'établissement volontaire dans l'industrie canadienne est soutenu par les autorités administratives, est mise en oeuvre par l'entremise du Service de collaboration ouvrière-patronale de la division des relations industrielles, du ministère fédéral du Travail.

Un comité mixte de production, qu'on désigne par les lettres CMP, est un conseil formé de représentants des travailleurs et de la direction d'une entreprise où les relations du travail sont régies par une convention collective intervenue entre un syndicat ou une autre organisation d'employés dûment reconnue par une Commission de relations ouvrières. Il s'occupe des questions d'intérêt mutuel autres que celles faisant l'objet des dispositions des conventions collectives: i.e. salaires, heures de travail, griefs, etc.

Les CMP sont de merveilleux moyens d'échange d'idées et d'informations sur les problèmes courants de l'entreprise. Ils se classent parmi les meilleurs modes d'expression de la démocratie industrielle. Leur efficacité repose sur l'éducation, la coopération et la discipline de ses membres, conditions essentielles à toute action démocratique. Institutions volontaires, elles sont de par leur nature des instruments plus efficaces que ceux imposés dans plusieurs pays où l'oppression est de règle.

Le gouvernement canadien encourage l'établissement de ces comités en raison de la contribution qu'ils peuvent apporter à l'économie du pays et à la paix industrielle. Le travail du Service de collaboration patronale-ouvrière est approuvé par les principales associations d'employeurs et d'employés y compris la Confédération des Travailleurs Catholiques du Canada. Le Service de collaboration patronale-ouvrière facilite l'établissement de CMP au sein des entreprises, avise les comités déjà établis qui recourent à ses services dans la solution de leurs problèmes, procure aux comités une variété de documents publicitaires nécessaires à leurs opérations et poursuit périodiquement des travaux de recherches sur les comités efficaces.

A l'heure actuelle, il y a au Canada 746 CMP établis d'après les recommandations du Service de collaboration patronale-ouvrière. Ils impliquent plus de 300,000 travailleurs. De ces 746 CMP, 153 sont dans la province de Québec.

Les Comités patronaux-ouvriers de production étaient très en faveur dans les pays démocratiques alliés pendant la Deuxième Grande Guerre. Le but principal des CMP était alors d'augmenter la production, afin de fournir aux forces armées ce dont elles avaient un pressant besoin. En fait, les CMP avaient été conçus comme des instruments de l'entreprise, pour solutionner les problèmes préjudiciables à la production: méthodes inefficaces de travail, gaspillage de matériel et de temps, accidents, absentéisme, éclairage défectueux et mauvaise ventilation, duplication d'efforts, outils endommagés, pour ne nommer que ceux-là. Les bons effets du CMP sur la production se sont répercutés, mais de façon moins perceptible, sur le moral des employés. A l'avènement de la paix on constatait déjà au'un CMP était plus qu'une aide à la production.

On se rend compte de plus en plus, par exemple, que la consultation conjointe est un moyen idéal et très efficace de se renseigner à tous les degrés sur les activités de la compagnie et de l'industrie et ce jusqu'aux points les plus éloignés d'une usine. 
I1 est maintenant établi qu'un CMP aide puịssamment à la propagation des intormations du haut vers le bas et permet une meilleure réception des idées et opinions émanant des rangs inférieurs. II est communément admis que la communication dans une entreprise n'est pas satisfaisante si elle fonctionne dans un seul sens, du haut vers le bas. L'expérience a démontré que les renseignements provenant du plus haut rang d'une organisation sous forme de directives, bulletins, lettres, pancartes, feuillets insérés dans les enveloppes de paie, bien qu'ils aient leur place dans un programme d'information, ne sont pas reçus par les employés aussi efficacement qu'ils devraient l'être.

Un officier de personnel d'une firme britannique, Howard Marshall, directeur des relations publiques et du personnel de l'établissement Richard Thomas and Baldwins, Ltd., a fait à ce propos le commentaire suivant: «Nous sommes toujours anxieux de communiquer aux autres ce que nous croyons qu'ils devraient savoir, sans réaliser que la véritable communication est comme un «pont» vers la compréhension mutuelle, et se résout en un effort conjoint vers un but commun. »

Le Comité mixte de production remplit ce rôle. Lorsque CMP fonctionne bien, le travailleur constate promptement que la direction, par l'entremise du comité, accueille et considère ses suggestions relatives au fonctionnement de l'usine, avec le résultat qu'il est conscient de n'être plus seulement un nom sur la liste de paie.

Réciproquement, la direction peut atteindre les travailleurs qui en viennent à concevoir les problèmes qu'elle doit affronter. D'après Stanley C. Allyn, directeur de la Chambre de Commerce des Etats-Unis, «Il ne suffit pas à la direction de connaître que les travailleurs sont informés de ce que les administrateurs pensent, il est également important que la, direction sache ce que les travailleurs pensent. 》

Par l'entremise d'un CMP, une compagnie peut aviser ses travailleurs de ses perspectives d'affaires, de son développement futur ou du réaménagement de son installation. Les changements sont toujours mieux acceptés lorsqu'ils sont annoncés d'avance; le contraire se produit lorsqu'ils sont effectués sans avis. Lorsque les travailleurs savent que leurs représentants ont participé aux délibérations qui ont amené tels changements, ils sont plus aptes à s'y conformer. A ce propos, Monsieur Marshall signale que: «La préparation des opinions, l'information préalable, l'explication qui aidera les employés à s'adapter aux nouvelles directives et aux situations non prévues et, ce qui est important, l'opportunité qui leur est donnée de participer à l'établissement de la politique, leurs propres commentaires et conseils, produits de leur expérience - toutes ces choses sont essentielles au travail d'équipe. Ce résultat sera atteint par toute entreprise qui saura mettre en oeuvre les multiples contributions de tous ses employés et qui, en même temps, démontrera' que celles-ci servent à une fin valable.»

Voici d'après le professeur Florence Peterson, directeur, Graduate Department of Social Economy, Bryn'Mawr College, quels ennuis peuvent résulter de l'omission à fournir en temps opportun des renseignements nécessaires: «L'introduction d'une machine nouvelle peut être rationnelle en autant qu'il s'agisse d'améliorer l'entreprise, mais il est également logique pour ceux qui auront $\dot{a}$ subir préjudice de la nouvelle méthode de protester contre son introduction. Une partie de l'agitation pourrait être atténuée si les travailleurs étaient bien informés de tous changements projetés et si l'assurance leur était donnée que la direction s'intéresse autant à leur avenir qu'aux possibilités de la nouvelle machine. » 
Que les travailleurs soient renseignés sur ce qui concerne la Compagnie pour laquelle ils travaillent, et ce autant que les actionnaires euxmêmes, est un postulat généralement accepté aujourd'hui. A la dernière convention annuelle de l'Association des Manufacturiers Canadiens, tenue récemment à Québec, le Sénateur Wallace F. Bennett, d'Utah, a rappelé avec à-propos qu'un employé doit connaître tout ce qui concerne son emploi, ainsi que la place qu'il occupe dans l'ensemble des opérations de la' Compagnie; ses buts et les moyens mis en oeuvre pour les réaliser. Ceux qui connaissent l'industrie savent fort bien que le travailleur qui est mis «au courant» est un individu plus satisfait - et, en même temps, beaucoup plus productif.

On constate aussi qu'un CMP permet de considérer les travailleurs comme des êtres humains qui ont un besoin naturel de s'exprimer et de se faire reconnaître personnellement, et qui désirent travailler comme associés et non comme supérieurs et subordonnés. En tant que relations humaines, les relations du travail sont vraiment bonnes lorsqu'elles sauvegardent la dignité du travailleur et stimulent son initiative.

Les CMP tendent également à favoriser la compréhension entre le capital et le travail que les Evêques de la province de Québec préconisent dans leur lettre pastorale. D'après Glen W. Cleeton, professeur de psychologie industrielle à Carnegie Institute of Technology, "le maintien de relations humaines harmonieuses dans l'industrie exige que la direction et les travailleurs comprennent réciproquement leurs points de vue. Les conflits collectifs peuvent être réduits par la promotion de la compréhension mutuelle».

Pendant longtemps, les facteurs humains dans l'industrie ont été négligés. La technologie a soulagé le travailleur de beaucoup de tâches ardues, mais elle a, en même temps, réduit son activité créatrice. Si bien que le travail, activité humaine pourtant normale, est souvent devenu terne et peu intéressant, sinon une frustration dans l'esprit des travailleurs. Le manque de considération envers les valeurs spirituelles du travail est responsable des situations où l'avarice domine, les tensions sont aigues et les conflits collectifs se rencontrent. Pour toucher au coeur du problème des relations humaines dans l'industrie, les employeurs et les travailleurs doivent trouver un terrain d'entente permettant le développement du respect mutuel des désirs et des nécessités de chacun.

Parmi les désirs des travailleurs, en plus de ses nécessités physiques, nous pouvons mentionner: le désir de s'exprimer, de réussir personnellement, et celui d'appartenir à un groupe. Comme être humain, le travailleur cherche à se maintenir en relation sociale avec ses employeurs et ses compagnons. Il répond favorablement lorsque ceux qui l'entourent concourent à son bien-être physique; lorsqu'il est reconnu comme un individu, un partenaire et non comme un serviteur; quand il est renseigné sur le résultat de ses efforts et sur les plans et politiques de la compagnie; enfin, quand son entourage lui accorde son amitié et son respect. Que l'une de ces conditions vienne à manquer, le travail d'équipe n'atteindra plus son maximum d'efficacité. Un ouvrier donne tout son rendement lorsqu'il met son coeur au travail, lorsqu'il est fier de travailler pour son employeur et qu'il se rend compte que sa participation contribue au succès de l'établissement.

Un comité patronal-ouvrier de production qui fonctionne normalement peut réaliser ces conditions et satisfaire aux désirs des travailleurs d'être considérés comme des individus, d'appartenir à un groupement et d'être renseignés sur leur occupation. Quand les travailleurs ont la liberté de soumettre leurs idées à la direction par l'entremise d'un CMP, 
et que leur représentant reçoit les confidences de la direction et participe à la solution des problèmes de la compagnie, ils constatent alors que leurs opinions sont considérées, que leurs travaux sont importants et qu'ils font tous partie d'une équipe qui coopère à la réussite de l'entreprise.

Parlant à la convention annuelle de l'Association des Manufacturiers Canadiens, tenue à Québec en juin, le Ministre fédéral du Travail, $\mathbf{M}$. Gregg, a déclaré que les comités mixtes de production «donnent aux travailleurs l'avantage de connaître la direction, qui cesse d'être un « elle » impersonnel sur qui peut être rejeté le blâme de tout ce qui irrite. On constate que le CMP développent la confiance mutuelle et contribuent pour beaucoup à éliminer la vieille idée de deux camps opposés, d'intérêts et de loyautés divergents.

L'existence d'un CMP dans une usine donne à tous l'occasion d'exposer les ennuis mineurs qui à la longue peuvent avoir un effet sérieux sur le moral s'ils ne sont pas corrigés.

Il ressort également que la coopération patronale-ouvrière a contribué à la découverte de talents inconnus et insoupçonnés parmi les employés qui, ajoutés à ceux des gérants professionnels et de leurs assistants techniques, ont multiplié pour autant les ressources créatrices de la compagnie.

La coopération entre la direction et les travailleurs par l'intermédiaire d'un CMP constitué d'après les recommandations du ministère fédéral du Travail n'amène aucune aliénation des droits, des responsabilités ou des libertés de l'une ou de l'autre des parties. Aucun groupe ne cède en rien. Les travailleurs n'essaient pas de se substituer à la direction; et la direction ne tente pas d'empiéter sur le territoire de l'union. Le comité remplit le rôle d'aviseur et il est laissé à la direction le soin de donner suite à ses recommandations. Basé sur une reconnaissance sincère des fonctions et des responsabilités de chacun, un CMP est, de fait, une mise en commun des connaissances des travailleurs et de la direction, de l'habileté, de l'expérience et des talents de chacun en vue d'aviser sur les problèmes de la compagnie qui les affectent mutuellement.

Clinton S. Goldsen, qui fut pendant longtemps vice-président international des Travailleurs Unis de l'Acier d'Amérique (United Steelworkers of America, CIO), et qui est maintenant conférencier invité sur des problèmes du travail à Harvard, a délimité en trois étapes l'évolution des organisations de travailleurs, de la «conspiration》 aux associations légitimes dûment constituées. $\mathrm{Au}$ premier stade, les travailleurs de métier ou d'industrie ont formé un mouvement de solidarité en vue de promouvoir l'adhésion à une nouvelle institution; au deuxième stade, l'organisation ouvrière a' demandé sa reconnaissance comme représentant autorisé des travailleurs individuels qui en font partie; et au troisième stade, l'union est acceptée par la direction comme une institution légitime et un participant constructif dans l'entreprise.

Plusieurs parmi les officiers de la direction et les représentants des unions croient que le simple fait de la certification établit la coopération et que la fin, plutôt qu'une étape de cette évolution progressive, a été atteinte. Ce qui voudrait dire que lorsque deux groupes puissants d'intérêts divergents ont réussi à établir un équilibre douteux, la tâche de rajustement et d'entente serait pratiquement complétée.

Bien que la convention collective contienne généralement les règles qui gouvernent les rapports mutuels entre la direction et les représentants de l'union des employés, elle n'établit pas automatiquement les conditions et l'atmosphère nécessaires à la coopération vraiment créative et constructive. Cette forme «d'armistice» n'apporte pas à la direction ou aux employés le sens de 
la sécurité durable, ni les satisfactions spirituelles et personnelles que les êtres humains espèrent retirer de leur travail et de leurs associations.

Par contre, un nombre croissant d'employeurs et de travailleurs n'acceptent pas l'opinion que la reconnaissance syndicale soit une forme de coopération et ne se contentent pas de l'équilibre instable de deux groupes sociaux ou professionnels apparemment hostiles. Ils admettent que les deux organisations d'intérêts différents sur lesquelles repose le fonctionnement de l'industrie moderne s'efforcent de co-exister dans une paix relative facilitant le rapprochement; ce fait représente pour eux une réalisation valable mais qui ne peut être considérée comme une fin ultime et désirable.

Ils veulent aller encore plus loin et promouvoir des relations industrielles harmonieuses constructives.
Ils croient, et l'expérience a confirmé leurs convictions, que chaque employé est un être humain dont la valeur potentielle est capable non seulement de contribuer avec avantage au succès de l'entreprise qui l'emploie, mais aussi au bonheur et au bien-être tant de ses compagnons de travail que de ses associés de la direction.

La troisième étape visée par $M$. Golden n'a pas encore été atteinte partout. De plus, en certains cas, la seconde n'a pas encore été réalisée. Mais lorsque la direction reconnaît l'association ouvrière comme une institution légitime, qui peut éventuellement devenir un participant constructif dans l'industrie, il semble que logiquement la prochaine étape soit celle de participer à l'organisation d'un comité patronal-ouvrier de production.

\title{
JURISPRUDENCE DU TRAVAIL
}

\author{
par Me JEAn-H. Gagné, \\ avocat et professeur à la Faculté des sciences sociales de Laval
}

Dans cette chronique qui paraîtra dans chaque numéro de la Revue des Relations Industrielles, nous présenterons les principaux points d'intérêt rencontrés dans les sentences arbitrales rendues par les conseils d'arbitrage de la Province de Québec, durant les trois mois précédant la parution de chacun des numéros de la Revue.

A l'occasion, nous analyserons aussi, sous cette rubrique, les décisions judiciaires rendues par les Cours de Droit commun durant la même période et pertinentes au Droit du travail. Les sentences arbitrales étudiées dans le présent numéro sont celles rendues durant les mois de mai, juin et juillet 1951. Nous ne rapportons que les sentences contenant des commentaires d'intérêt particulier pour nos lecteurs.

1-Regent Knitting Mills Limited, StJÉRÔME ET L'UNION DE SES EMPLOYÉS.

Mandat des arbitres: le boni de vie chère et la période de revision de l'indice du coût de la vie.

Dans une sentence unanime, le tribunal recommande que le boni de vie chère soit réparti sur le nombre d'heures réellement travaillées par les employés; que l'indice du coût de la vie soit revisé à tous les trois mois et que, à la hausse comme à la baisse, l'on en tienne compte dans les réajustements de salaires.
(Union fédérale des employés du textile de St-Jérôme, local 254; nature de l'entreprise: fabrique de textile secondaire; 800 employés concernés sur 1,104; union affiliée au C.M.T.C.; sentence unanime rendue le 10 mai 1951; membres du tribunal: arbitre patronal: Me Lucien Thinel, arbitre syndical: Me Maurice Fortier, président: Me Roger Lacoste.)

2-Besner Building, Montréal, et L'UNION DE SES EMPLOYÉS. 